\title{
Péritonite et pneumopéritoine au décours d'une remise en place d'une sonde de gastrostomie
}

\author{
Peritonitis and Pneumoperitoneum after Gastrostomy Tube Replacement
}

\author{
S. Beroud - G. Passot $\cdot$ P. Rousset \\ Reçu le 6 novembre 2018; accepté le 3 janvier 2019 \\ (C) SFMU et Lavoisier SAS 2019
}

\section{Introduction}

Les alimentations sur gastro/jéjunotomies se sont considérablement développées ces dernières décennies. On estime qu'environ un tiers des patients avec une stomie d'alimentation présenteront au cours de leur vie un épisode d'arrachement/malposition (volontaire ou non) de leur sonde [1]. Ces situations peuvent amener des patients à consulter aux urgences pour mise en place d'une nouvelle sonde et les exposent à des complications de manipulations potentiellement graves qu'il faut connaître pour les éviter.

\section{Observation}

Une patiente âgée de 68 ans, en hospitalisation à domicile (HAD), est adressée aux urgences par son infirmière devant une asthénie fébrile. Elle présentait comme principal anté-

\footnotetext{
S. Beroud $(\triangle)$

Service d'accueil des urgences,

centre hospitalo-universitaire de Lyon-Sud, hospices civils de Lyon, 3, quai des Célestins, F-69002 Lyon, France

e-mail : sebastien.beroud@chu-lyon.fr

G. Passot

Service de chirurgie digestive, unité chirurgicale d'urgence, centre hospitalo-universitaire de Lyon-Sud, hospices civils de Lyon, 3, quai des Célestins, F-69002 Lyon, France

\section{P. Rousset}

Service d'imagerie médicale et interventionnelle, centre hospitalo-universitaire de Lyon-Sud, hospices civils de Lyon, 3, quai des Célestins, F-69002 Lyon, France

Faculté de médecine Lyon-Sud-Charles-Mérieux, université Claude-Bernard-Lyon-I,

165, chemin du Petit-Revoyet, BP 12,

F-69921 Oullins cedex, France
}

cédent un accident vasculaire cérébral (AVC) hémorragique datant de neuf ans avec de lourdes séquelles (patiente grabataire avec hémiplégie gauche, aphasie et troubles vésicaux). Devant des troubles de déglutition majeurs séquellaires, responsables d'une dénutrition et de pneumopathies d'inhalation à répétition, une gastrostomie pour nutrition entérale fut réalisée dans les mois qui ont suivi l'AVC. Les paramètres vitaux à l'admission retrouvaient une tachycardie à 146/ minute, une tension artérielle à $105 / 61 \mathrm{mmHg}$, une saturation à $96 \%$ en air ambiant, une température à $37,3^{\circ} \mathrm{C}$ et un score de Glasgow à 11 . Son état de conscience était semblable à son état de base constaté lors de précédents passages aux urgences. L'interrogatoire était non informatif du fait des troubles de conscience persistants et des troubles phasiques. L'examen montrait une tachypnée avec une auscultation pulmonaire peu contributive ainsi qu'une défense abdominale en hypocondre et flanc droit. La biologie révélait un syndrome inflammatoire avec une protéine $\mathrm{C}$ réactive à $380 \mathrm{mg} / \mathrm{l}$ et des leucocytes à $20 \mathrm{G} / 1$ (dont $85 \%$ de polynucléaires neutrophiles) sans anomalie du bilan hépatopancréatique. Le ionogramme sanguin était normal et la fonction rénale correcte. La patiente était anurique depuis son admission. Devant ce tableau de douleur abdominale fébrile, un scanner abdominopelvien injecté est réalisé et retrouve un pneumopéritoine avec péritonite sur malposition de la sonde de gastrostomie qui est retrouvée en position intrapéritonéale (Fig. 1). Le classeur de l'HAD indiquait que la patiente avait arraché sa sonde de gastrostomie l'avant-veille. Une sonde de Foley avait été mise en place en solution de dépannage par l'infirmière, sans contrôle particulier. Médicaments et nutrition entérale ont été administrés par cette sonde. Après concertation collégiale justifiée devant les comorbidités de la patiente et la gravité de la présentation clinique, une chirurgie en urgence est décidée. Un lavage de la cavité péritonéale est réalisé sous cœlioscopie ; l'ancien orifice de gastrostomie n'étant pas retrouvé, un nouvel orifice est créé en fin d'intervention avec mise en place d'une nouvelle sonde de gastrostomie. Les suites postopératoires immédiates seront simples. 

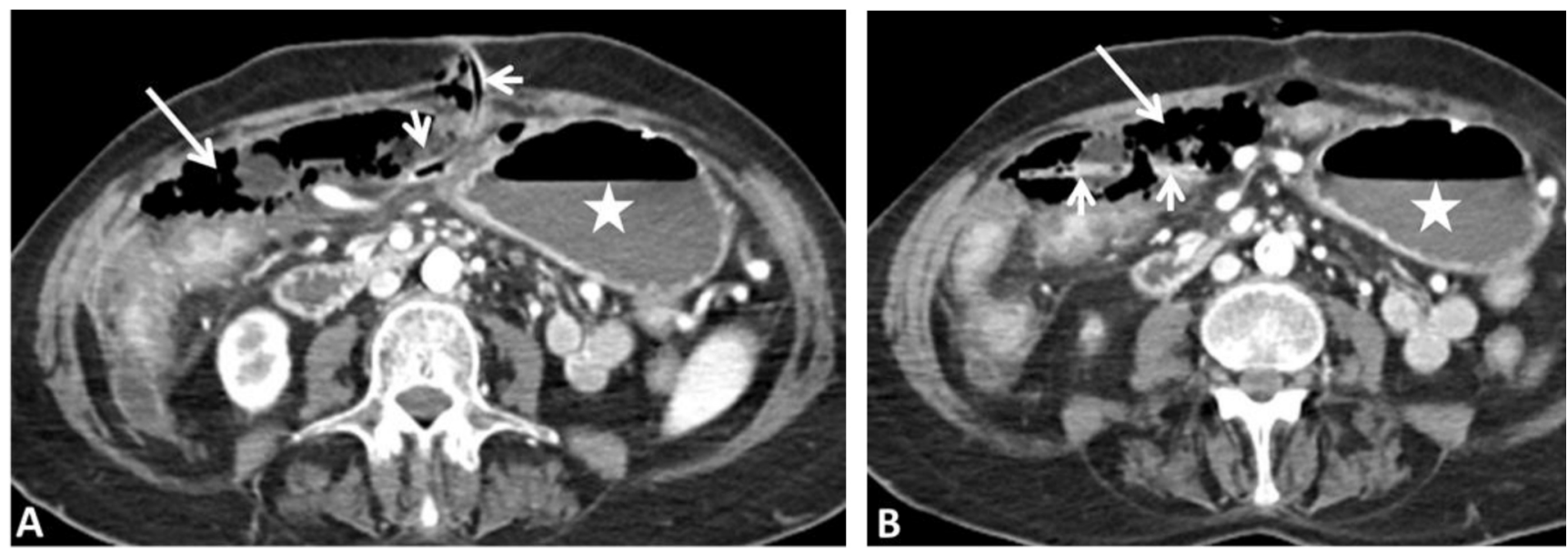

Fig. 1 Coupes tomodensitométriques axiales du haut (A) vers le bas (B) montrant le trajet extragastrique de la sonde de gastrostomie (flèches courtes) avec la présence d'un pneumopéritoine (flèches longues) et d'une péritonite en regard du trajet intrapéritonéal de la sonde. Étoiles $=$ estomac

La patiente effectuera un séjour en gériatrie avant de regagner son domicile en HAD.

\section{Discussion}

Dans les situations d'arrachement de sonde de gastro/jéjunostomie, la priorité est au repositionnement en urgence d'une nouvelle sonde. En effet, l'orifice de stomie peut se refermer dans des délais très courts, même s'il est ancien, rendant parfois nécessaire une procédure endoscopique pour mise en place d'une nouvelle sonde. En cas d'orifice mature ( $>30$ jours), une sonde de Foley doit être mise en place de manière transitoire. En revanche, en cas d'orifice non mature $(<30$ jours $)$, il ne faut pas tenter de repositionner une sonde à l'aveugle mais demander un avis spécialisé en urgence (chirurgien viscéral ou gastroentérologue interventionnel). Les complications lors d'une remise en place en aveugle d'une sonde de gastrostomie ne sont pas rares, avec 3 à $6 \%$ de complications dont $10 \%$ sont létales [2-4]. Les populations préférentiellement concernées par ce type d'incidents sont susceptibles, du fait de terrains particuliers (démence, séquelles de traumatisme crânien sévère ou d'AVC avec troubles de déglutition séquellaires, paralysie cérébrale, etc.), d'avoir une présentation atypique. Ces patients peuvent ne pas réagir en cas d'insertion péritonéale (faux trajet) ou lors de l'instillation de liquide du fait d'une réponse altérée à la douleur. Même en présence d'un orifice stomial mature, un mauvais positionnement de la sonde reste possible lors de son remplacement. Au moindre doute sur une insertion anormale (geste douloureux, sensation de résistance à l'insertion douce, absence de borborygme lors de l'insufflation d'air ou douleur lors de l'instillation de sérum physiologique), un contrôle du positionnement est nécessaire [2,5]. Des auteurs ont proposé des protocoles pour limiter la iatrogénie lors du remplacement de sonde [6], mais leur application permet seulement de diminuer le risque de complication, sans l'annihiler [7]. De plus, certains protocoles ne sont valides que lors d'un changement avec une sonde initiale encore en place [6]. Des auteurs prônent la réalisation systématique d'un contrôle de positionnement de la sonde avant utilisation soit par voie endoscopique, soit radiologiquement avec injection de produit de contraste dans la stomie (gastrografine) [5,8]. Le contrôle radiologique est moins chronophage, moins coûteux et a l'avantage de ne pas reposer sur la disponibilité du plateau d'endoscopie digestive, le rendant plus accessible. Ces contrôles éviteraient de se retrouver dans une situation telle que celle décrite dans notre cas, d'autant que si notre patiente a pu retrouver son état basal, plusieurs décès ont déjà été rapportés [2-4].

\section{Conclusion}

La remise en place d'une sonde de gastro/jéjunostomie est une urgence du fait du risque de fermeture rapide de l'orifice stomial. Ce geste, réalisable par l'urgentiste en cas d'orifice mature, bien qu'assez simple de réalisation et rapide dans son exécution, doit être réalisé dans de bonnes conditions. À l'instar d'autres auteurs, on propose comme attitude de sécurité un contrôle systématique du bon positionnement de la sonde avant utilisation afin d'éviter des complications iatrogènes.

Conflit d'intérêt : les auteurs déclarent ne pas avoir de lien d'intérêt. 


\section{Références}

1. Shahbani DK, Goldberg R (2000) Peritonitis after gastrostomy tube replacement in the emergency department. J Emerg Med 18:45-6

2. Platt MS, Roe DC (2000) Complications following insertion and replacement of percutaneous endoscopic gastrostomy (PEG) tubes. J Forensic Sci 45:833-5

3. Triantafyllou K, Papanikolaou IS, Stasinos I, et al (2014) Percutaneous endoscopic gastrostomy tube replacement unexpected serious events. Nutr Clin Pract 29:142-5

4. Taheri MR, Singh H, Duerksen DR (2011) Peritonitis after gastrostomy tube replacement: a case series and review of literature. JPEN J Parenter Enteral Nutr 35:56-60
5. Lohsiriwat V (2013) Percutaneous endoscopic gastrostomy tube replacement: a simple procedure? World J Gastrointest Endosc $5: 14-8$

6. Suzuki Y, Urashima M, Yoshida H, et al (2009) The sky blue method as a screening test to detect misplacement of percutaneous endoscopic gastrostomy tube at exchange. Intern Med 48: 2077-81

7. Abbott L, Bromberek EF, Isserman JD, et al (2018) Tube feed malposition and associated peritonitis. J Emerg Med 55:e107-8

8. Nishiwaki S, Araki H, Fang JC, et al (2011) Retrospective analyses of complications associated with transcutaneous replacement of percutaneous gastrostomy and jejunostomy feeding devices. Gastrointest Endosc 74:784-91 